\title{
Certain Investigation on Healthcare Monitoring for Enhancing Data Transmission in WSN
}

\author{
Senthil Kumar Swami Durai ${ }^{1} \cdot$ Balaganesh Duraisamy $^{2} \cdot$ J.T. Thirukrishna ${ }^{3}$
}

Received: 19 April 2021 / Revised: 9 July 2021 / Accepted: 8 August 2021 / Published online: 24 August 2021

(c) The Author(s), under exclusive licence to Springer Science+Business Media, LLC, part of Springer Nature 2021

\begin{abstract}
Wireless Sensor Networks are often to perform autonomous sensing and controlling the real world objects through the sensor nodes across the globe. Since these sensor nodes are operated by the energy of the battery that has been performed a vital role in deploying a sensor network. Hence, the battery power needs to be minimized to prolong network lifetime for healthcare applications. The monitored data transmission is very important to process in building wireless sensor networks. In order to provide efficient data transmission wireless technology standards are followed as IEEE 802.15.4 standards that provide desirable communication between end to end with optimal routes using the proposed Energy Optimization Algorithm. The proposed algorithm has been improved the data packet transmission efficiency up to 25\% and also helps to prolong the life time of Wireless Sensor Networks in order to achieve the efficient data transmission for health care monitoring.
\end{abstract}

Keywords Wireless sensor networks · Energy · IEEE 802.15.4 standard · Sensor node · Power consumption · Zigbee · LabVIEW

\section{Introduction}

Earlier the sensor network consists of little sensor nodes that were linked to the central processing system through a wire. But the focus is gradually changed onto wireless and scattered nodes employed for sensing information. The wireless environment is scattered and wireless because the precise location of the particular event will not be known where the scattered sensing permits for nearer positioning to the incident, unlike a single sensor node. Many areas using the WSN applications require a number of nodes for overcoming the environmental barriers like a line of sight. It must also

Senthil Kumar Swami Durai

harisen1234@yahoo.co.in

Balaganesh Duraisamy

balaganesh@lincoln.edu.my

J. T. Thirukrishna

maill2thiru@gmail.com

1 Post Doctoral Fellowship, Department of CSE, Lincoln University College, Selangor Darul Ehsan, Malaysia

2 Faculty of Computer Science and Multimedia, Selangor Darul Ehsan, Malaysia

3 Department of ISE, Dayananda Sagar Academy of Technology and Management, Bangalore, India be noted that the environment subjected to observation does not possess a planned structure either for energy nor relays. It becomes mandatory for the sensor nodes to endure on tiny, limited power and relay through a wireless medium $[1,2]$.

The major requirements of the sensor networks are its scattered processing abilities. This is quite necessary for sensor nodes because the communication itself devour energy from the wireless network nodes. A static system governed by administrator reveals that the sensors require communicating over a large distance thus depleting most of the network energy [3, 4]. It will be better to process all the information close by for reducing the number of information to be communicated.

Medical observations are performed in two ways as wearable devices that could be possibly mounted on to the surface of the human body or these medical devices could be inserted into the body of humans [5,6]. Other than these applications they are employed for measuring the positions of the body, tracking location of a person, entire observation of sick patients in hospitals and at home. The body area networks are used to gather information about a patient's health condition, strength and energy outlays [7, 8].

The wireless sensor networks are employed for monitoring the conditions of machines because they provide notable 
cost reductions. These sensors could be placed in locations which are impossible for the reach of humans [9].

The sensor nodes are battery operated and its maintenance is difficult since the battery cannot be recharged nor replaced. Certain techniques are required for conserving the energy and equalizing the loads during the design and deployment phase of WSNs [10, 11].

The majority of sensor nodes are positioned in regions without any proper planning or infrastructure. The common way to position the nodes within dense areas would be throwing them from the air $[12,13]$. During these situations, the nodes must be capable enough to explore its connection and allotment. The wireless sensor networks are used for identifying even small activities of soil and alteration in numerous parameters happening very before the avalanche. Based on the accumulated data it is probable to identify the incidence of avalanche very before it actually happens [14, 15].

Reducing the energy utilized for communication in wireless sensor networks with the use of effective clustering. With clustering, the problems might arise due to increased cluster heads their allotment within the network for making sure that minimal energy is consumed for network operations. The conventional clustering technique deals with the cluster heads and does not guarantee network coverage within the network [16]. The authors proposed an optimal clustering technique for restricting the energy utilization and offering better coverage. The experimental results reveal that the proposed technique overcomes the conventional clustering technique and enhances the performance of the network [17].

Wireless sensor networks employ grouping techniques for addressing issues in network lifetime and energy conservation among the sensor nodes [18]. The authors focused on addressing the problem with existing methods by proposing a method to enhance the lifetime of the network. The technique constantly chooses a head for the groups and the node with highest remaining energy is chosen. The experimental results reveal that the proposed technique conserves energy greatly by equalizing the energy consumption by all the sensor nodes and prolongs the network lifetime as evaluates with other conventional grouping protocols [19].

\section{Related Work}

Remote monitoring framework for supervising various metrics such as Electrocardiogram, pulse rate, $\mathrm{SpO} 2$, respiratory rate, and temperature has been designed in [20]. The data from the sensors is obtained and processed by microcontrollers, the processed information is delivered to smartphone through Wi-Fi module. The application rises an auto alarm when abnormal values are detected in patient's data and delivers an alert signal to the professional's software via 3G mobile network and Wi-Fi.

Wireless Body Area Sensor Network built using ZigBee module is proposed in [21]. Consisting of a series of biosensors connected to a body of patient. A wireless router is used to relay the records to a wireless transceiver attached to a local control unit and chosen LabVIEW software because it has powerful DAQ. The collected data is carried out using Data Acquisition Framework (DAQ) provided by the National Instrument. So, the performance data produced by the LabVIEW software procured quickly.

A BSN architecture intended for continuous healthcare tracking using biosensors has been developed in [22]. A number of wireless biosensors were devised with the BSN architecture, including ECG and Blood oxygen saturation, Context Sensors such as accelerometers, humidity sensors, temperature and are also combined with the BSN cluster to assist the amalgamation of gathered data. In addition, a lightweight flash BSN card is designed for PDA, where the PDA will collect, analyse, display sensor signals can also act as router connecting the BSN clusters and host computer, rather than serving as processor it can collect sensor data and transmits for extended storage and pattern discovery through Wi-Fi/GRPS network.

A real-time, portable wireless remote monitoring framework was developed [23]. The framework tracks and control Heart rate and oxygen saturation in the blood of patients using ZigBee wireless technology, the pulse oximetry data are transmitted to database computer server in the area of WPAN. The sensor modules were developed with a framework for low energy which can configure energy consumption according to power source and current power operation scenarios. In the author presents the state of the art of different forms of the network of WBASN, their Networking mechanisms, applications of WBASN program design frameworks, privacy problems and protocols for power efficient networking. By means of some analysis about existing radio technology for such kind of network, the author has covered the latest developments. The author has also outlined numerous factors and problems of WBASN, possible visualizations and obstacles in this field are addressed [24].

Wireless sensor networks (WSN) play a key role in such a monitoring FRAMEWORK application, owing to the fact that WSN can give several advantages over other types of wireless frameworks in particular their scalability, improved productivity, power management, cost efficiency and design versatility. Wearable devices are contributing in a significant manner to disease prevention, diagnosis, and precautionary steps. As Wireless technology and healthcare advance, they will become more sophisticated with enhanced patient safety [25]. Advancements in sensor technology, the pervasive 
availability of cellular technology, and falling costs of embedded communication devices are opening up new mechanisms for improving patient care and quality of life. The rapid development of wearable bio sensors is changing the way we gather and interpret the information.

Although number of radical achievements have been noticed in the medical service sector over the last few years [26]. Adhering to an excellent standard of measure cause-ofdeath data remains crucial for improving health and decrease preventable deaths in every country. This paper presents ultimately to build a Remote health monitoring framework, that has potential to detect the specific abnormality of cardiac function, measure the temperature of individuals and measure the pulse rate. Furthermore, it ensures to notify the warning messages namely short message service (SMS) to the healthcare personnel in case of emergency.

The process of identifying and detecting COVID virus has become more importance all-around the world for some months. COVID virus has taken the first place for spreading so fast that has become hard to control. COVID has become so hard for detecting as the person are not showing symptoms immediately. Thus it is more important to find new methods to differentiate the COVID positive people with normal people to eliminate the possibility. Artificial Learning can be used to examine a person for COVID-19 as an alternative to traditional time-consuming and expensive methods [27].

Even though there are many papers on COVID virus, this paper is focused on detecting COVID virus using Artificial Learning classification techniques using X-ray pictures and predict the people is positive to COVID virus or not. Several research areas have implemented Artificial Intelligence. One of the most advantages of AI is that they are often implemented during a trained model to classify unseen images. In this study, Artificial Intelligence was used to detect whether a patient is positive for COVID-virus by analysing their lungs X-ray pictures [28]. Artificial intelligence can also be used to predict the status of person like he is positive to COVID or not by using existing evidence. Thus, predicting possibilities within the immediate future can help authorities to adopt the required measures.

\section{Energy Optimization Algorithm}

An Energy Optimization Algorithm proposed that adjusts transmission power with respect to the dynamic and timevarying channel characteristics. The EOA is the modified version of the adaptive power control algorithm, but the power allocation strategy is different in both. The wireless mote platform provides a $256 \mathrm{kbps}$, IEEE 802.15.4 wireless radio, 8 Channel A/D and an $8 \mathrm{MHz}$ microcontroller for on board digital signal processing. In node to node data transmission mesh topology setup is used.

The necessity of healthcare application used in proposed model, proposed algorithm supports efficient and fast data transmission between the Human body and receiver end. So it is much suitable to use in health care applications.

Attributes used in the Energy Optimization Algorithm is followed by equation,

Find received signal strength average (Rx) is given by, Where $\mathrm{Rx}$ is the weighted sum of received signal strength,

$R x=$ Relow $+(1-\delta 1) \times R x$

$R x=$ Relow $+(1-\delta 2) \times R x$

Relow is received signal strength lowest sample.

Then find the transmission range using differential calculation,

$\Delta p=\left\{\begin{array}{c|lc}3 & \text { if } & R x<T H R l \\ -2 & \text { if } & R x>T H R h \\ 0 & \text { if } & T H R l<\mathrm{Rx}<\mathrm{THRh}\end{array}\right.$

To calculate the threshold value,

$\mathrm{THRh}=\mathrm{THR} 1+\sigma$

Then, calculate the average weight of channel to compute the transmission power followed by equation,

$\sigma=\sqrt{ } 1 / n \sum_{k=1}^{n}(\mathrm{Rk}-\mathrm{Rx}) \mathrm{k}=1,2,3, \ldots . . \mathrm{n}$

In equations terms are mentioned as follows,

Relow-Received signal strength identifier in low.

Rate, Rx -Received signal strength average, $\sigma 1, \sigma 2-$ Weight average, THRl-Threshold value is low, THRhThreshold value is high, $\mathrm{n}, \mathrm{Rk}$ and $\mathrm{k}$-denotes Standard Deviations.

The above computation helps to increase the signal strength from the receiver end.

The proposed algorithm of Energy Optimization Algorithm, the equations are derived based on the signal strength computation. Received Signal Strength Identifier(RSSI) is should be greater in terms of power in decibels. Based on Eqs. 1, 2, 3, 4 and 5 the signal strength levels (High and Low) identified efficiently.

\subsection{Architecture of Energy Optimization Algorithm}

Various applications such as security and surveillance, Environmental monitoring, healthcare and wild life habitat monitoring information sends to base station. When 


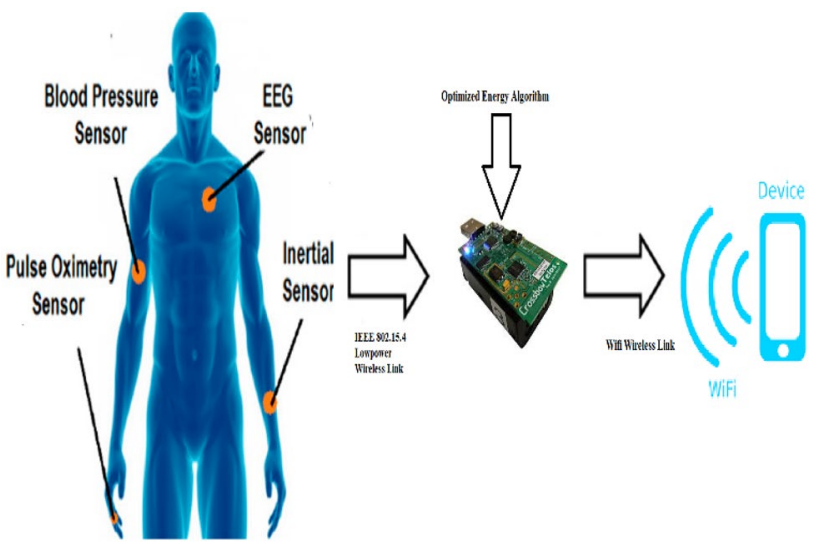

Fig. 1 Architecture of energy optimization algorithm

the information gathers from sensor, then it sends to IEEE 802.15.4 wireless device which has been used Energy Optimization Algorithm to transmit the data to mobile device by using Bluetooth technology as shown in the Figs. 1 and 2.

The significant of enhancement is about, Data transmission between human and base station in which where the human body goes abnormal condition then immediately data transmits to the corresponding authority to make correct decisions.

\subsection{Flow Diagram of Energy Optimization Algorithm}

The proposed algorithm flow diagram depicts that, initially data gathering has been done by sensor node. Then data packet transmission started to transmit the data using Energy Optimization Algorithm. Next step received data packets are calculated with measuring the average Received Signal Strength. If the signal strength measurement is low, then it's back to the data packets calculation otherwise Received Signal Strength Identifier target computes the transmission power and transmit the data to the base station when human body occurs abnormal conditions.

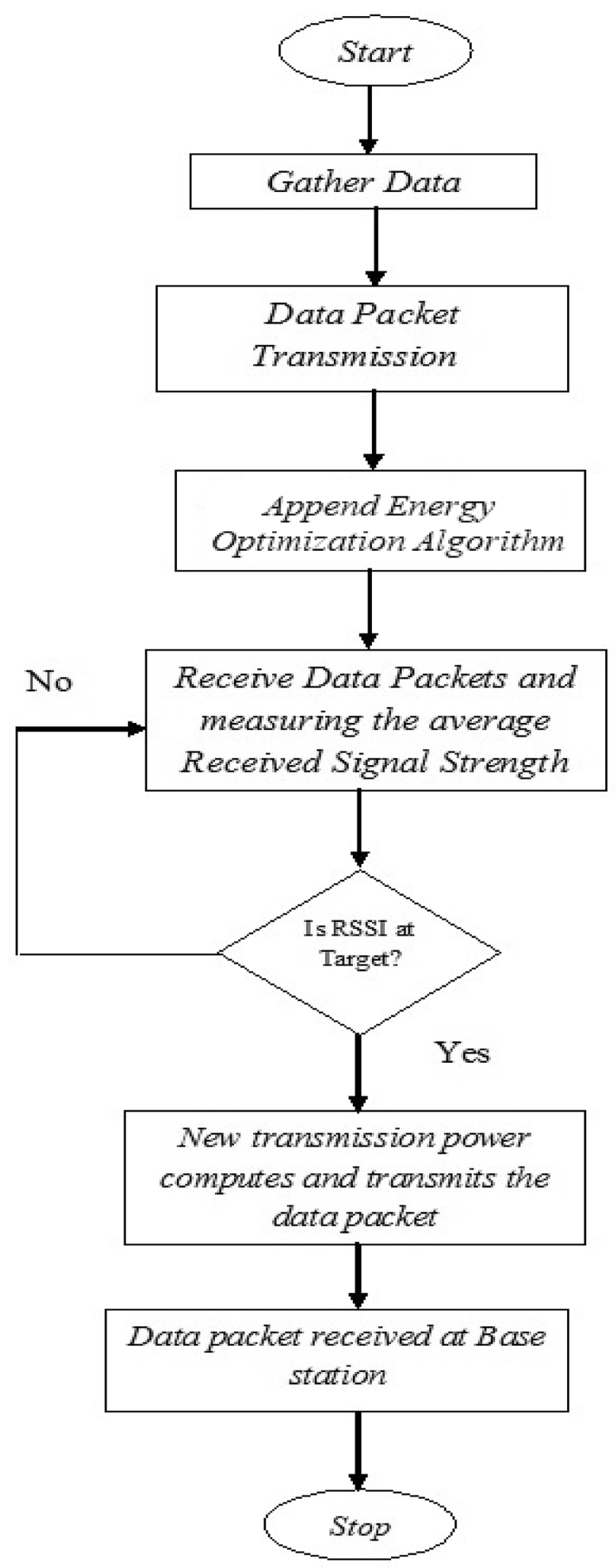

Fig. 2 Energy optimization algorithm flow diagram 


\subsection{Algorithm for Energy Optimization Algorithm}

Step 1: Identify the latest Received signal strength identifier sample.

// Categorize the received signal sample either high or low signal strength

Step 2: Identify the lowest Received signal strength identifier sample.

// Once low signal strength sample is identified then calculate the signal strength to increase the signal power.

Step 3: Rx: Average of Received signal strength identifier

// Receiver must able to know the average of Received signal strength for signal propagation.

Step 4: Rtt: Target of Received signal strength identifier

// Once signal strength more than signal target is identified

Step 5: if latest Received signal strength identifier $>\mathrm{Rx}$

/IIf the receiver identifies the latest signal strength the that must be greater than received one

Step 6: $R x=$ Relow $+(1-\delta 1) \times R x$

//To calculate the received signal strength, received signal strength identifier in low rate plus sender signal multiply with RSSI

Step 7: if latest Received signal strength identifier $<\mathrm{Rx}$

I/if the received signal strength is low than compute again

Step 8: $R x=$ Relow $+(1-\delta 2) \times R x$

//Once again receiver signal calculation done for improving signal strength.

Step 9: end if

//Once identified the signal strength RSSI stops the calculation

Step 10: end //Stop the transmission
The main objective of the MATLAB Simulation usage is to deploy Sensor network and increase the transmission power efficiency using Energy Optimization Algorithm.

Table 1 contains the information about various parameter and value used for simulation setup. The parameters are carrier frequency, power levels, Transmit Power Maximum, Transmit Power Minimum time, data frame size and data rate. Different values are assigned to transmit the data in efficient manner. The minimum range of Threshold value used 0.006 Watts i.e. -30 Decibels. The frequency range can be implemented Giga Hertz (GHz).

Yes, specified the area size of the deployed Sensor Networks needs to specified $100 * 100$ Meter. The range of channel frequency is $2.4 \mathrm{GHz}$ Bands and bandwidth $50 \mathrm{~Hz}$ used in proposed model. The transmit power range specified 0 to $-30 \mathrm{dBm}$.

\section{Result and Implementation}

Figure 3 shown, How the Energy Optimization Algorithm provides high speed transmission in efficient manner with low data rate while compared with Adaptive Transmission Power Control and State Based Adaptive Control Algorithm's. Y-axis mentioned as Transmission power in decibels and $\mathrm{X}$-axis referred as time in seconds. The transmission power utilization up to $25 \mathrm{dBm}$.

The proposed model of Energy Optimization Algorithm is compared with existing protocols such as Adaptive Transmission Power Control Algorithm and State Based Adaptive Control Algorithm. Proposed methodology comparatively competent with producing less transmission power utilization.

Figure 4 shown, How the Energy Optimization Algorithm provides high speed transmission in efficient manner with low data rate while compared with State Based Adaptive Control Algorithms. Y-axis mentioned as Transmission power in decibels and $\mathrm{X}$-axis referred as time in seconds. The transmission power utilization up to $25 \mathrm{dBm}$.

Figure 5 depicts the transmission power takes low power with higher efficiency that increased the power efficiency when compared Energy Optimization Algorithm with State Based Adaptive Power Control Algorithms, ESA provides good throughput. Y-axis mentioned as Transmission power in decibels and $\mathrm{X}$-axis referred as time in seconds. The transmission power utilization up to $25 \mathrm{dBm}$.

Figure 6 depicts the transmission power takes low power with higher efficiency that increased the power efficiency when compared Energy Optimization Algorithm with Adaptive Transmission Power Control, ESA provides good. The transmission power utilization up to $25 \mathrm{dBm}$ with throughput. Y-axis mentioned as Transmission power in decibels and $\mathrm{X}$-axis referred as time in seconds. Throughput evaluation is 
Table 1 Simulation parameters

\begin{tabular}{ll}
\hline Parameter & Value \\
\hline Carrier frequency & $2.4 \mathrm{GHZ}$ \\
Power levels(dBs) & $\{-30,-25$, \\
& $-15,-10,-$ \\
& $5,0\}$ \\
Transmit power maximum & $-30 \mathrm{dBm}$ \\
Transmit power minimum & $0 \mathrm{dBm}$ \\
Time & $100 \mathrm{~ms}$ \\
Data frame size & $100 \mathrm{bytes}$ \\
Data rate & $256 \mathrm{kbps}$ \\
\hline
\end{tabular}

estimated in the system model based on data transmission

Trănšmisssión Power Utilization

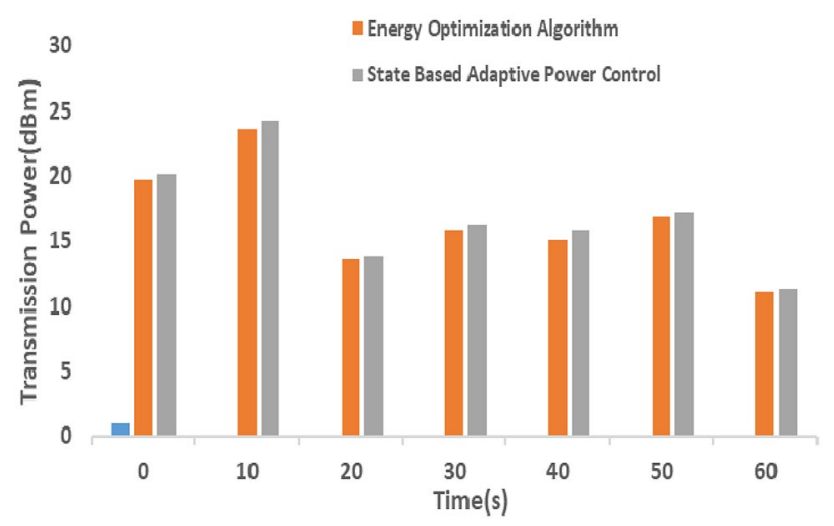

Fig. 3 Transmission power utilization comparison of adaptive transmission power control with energy optimization algorithm with respect to traffic load

\section{Transmission Power Utilization}

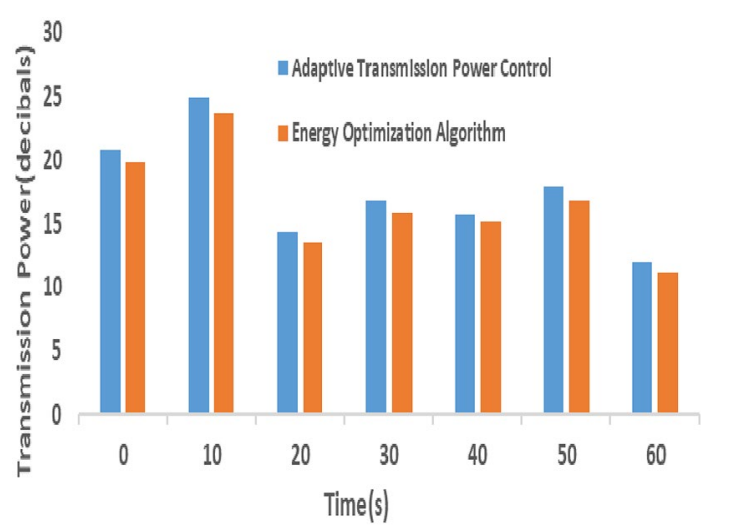

Fig. 4 Transmission power utilization comparison of state based adaptive control algorithms with energy optimization algorithm respect to no traffic load

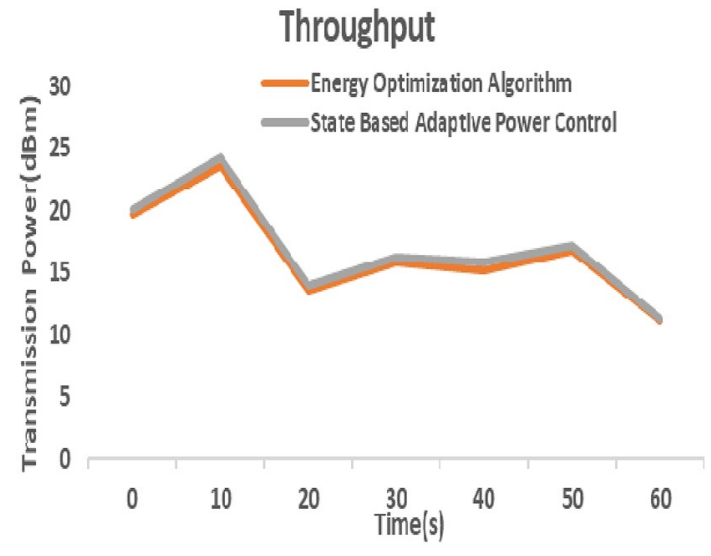

Fig. 5 Throughput comparison with respect to bandwidth

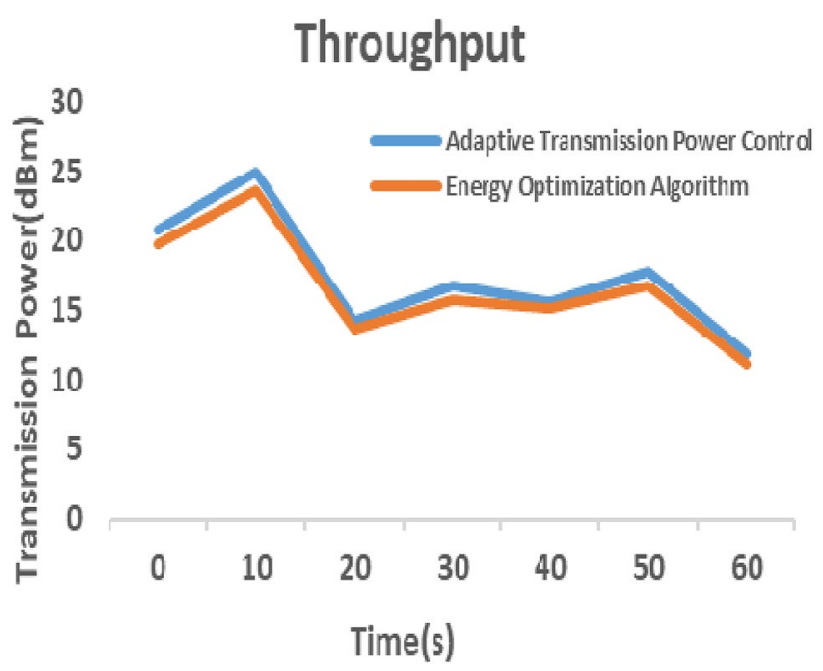

Fig. 6 Throughput comparison with respect to not using bandwidth

how the power utilization without and lapse between human and receiver.

\section{Conclusion}

Transmission range adjustment and the corresponding power level affect the performance of the sensor network when choosing the high power transmission. The proposed Energy Optimization Algorithm shown that data packet transmission efficiency is improved by $25 \%$ when compared to Adaptive Transmission Power control and State based adaptive power control algorithms. Using the Energy Optimization Algorithm increasing the power efficiency and also data 
transmission speed has been increased and battery consumed with less power. Based on the above result shown in the Fig. 4, Proposed algorithm supports in efficient way to increase the power efficiency that is used in health care application. In future extension is to identify the security based data transmission in Wireless Sensor Networks for health care monitoring.

\section{References}

1. X. Liu and W. Du, Energy-efficiency-based power allocation scheme in multi-user single-DF-relay cognitive networks, Wireless Personal Communications, Vol. 114, pp. 1927-1941, 2020. https://doi.org/10.1007/s11277-020-07455-3.

2. P. Kasyoka, M. Kimwele and S. M. Angolo, Towards an efficient certificateless access control scheme for wireless body area networks, Wireless Personal Communications, Vol. 115, pp. 12571275, 2020. https://doi.org/10.1007/s11277-020-07621-7.

3. M. Nabil and M. M. A. Faisal, Design, simulation and analysis of a high gain small size array antenna for $5 \mathrm{G}$ wireless communication, Wireless Personal Communications, Vol. 116, pp. 2761-2776, 2021. https://doi.org/10.1007/s11277-020-07819-9.

4. N. N. Joshi and R. D. Joshi, Energy conservation in MANET using variable range location aided routing protocol, International Journal of Wireless \& Mobile Networks, Vol. 3, No. 5, pp. 265, 2011.

5. S. K. S. Durai, B. Duraisamy and J. T. Thirukrishna, A novel approach for QoS enhancement with revision scheme using SeDSR protocol in wireless sensor networks, Wireless Personal Communications, 2021. https://doi.org/10.1007/ s11277-021-08466-4.

6. A. Farouk, A. Alahmadi, S. Ghose and A. Mashatan, Blockchain platform for industrial healthcare: vision and future opportunities, Computer Communications, Vol. 154, pp. 223-235, 2020. https:// doi.org/10.1016/j.comcom.2020.02.058.

7. F. Ingelrest, N. Mitton and D. Simplot Ryl, A turnover based adaptive hello protocol for mobile ad hoc and sensor networks, Fifteenth IEEE International Symposium on Modeling, Analysis, and Simulation of Computer and Telecommunications Systems (MASCOTS`07), pp. 9-14, 2007.

8. H. Yang and X. Wang, ECOCS: energy consumption optimized compressive sensing in group sensor networks, Computer Networks, Vol. 1469, pp. 159-166, 2018.

9. D. T. Tchakonté, E. Simeu and M. Tchuente, Lifetime optimization of wireless sensor networks with sleep mode energy consumption of sensor nodes, Wireless Network, Vol. 26, pp. 91-100, 2020. https://doi.org/10.1007/s11276-018-1783-3.

10. J. T. Thirukrishna, S. Karthik and V. P. Arunachalam, Revamp energy efficiency in homogeneous wireless sensor networks using Optimized Radio Energy Algorithm and Power-aware Distance Source Routing protocol, Future Generation Computer System, Vol. 81, pp. 331-339, 2018. https://doi.org/10.1016/j.future.2017. 11.042 .

11. W. K. Lai, S. Y. Hsiao and Y. C. Lin, Adaptive backup routing for ad-hoc networks, Computer Communications, Vol. 30, No. 2, pp. 453-464, 2007.

12. M. Z. Uddin, M. M. Hassan, A. Alsanad and C. Savaglio, A body sensor data fusion and deep recurrent neural network-based behavior recognition approach for robust healthcare, Information Fusion, Vol. 55, pp. 105-115, 2020. https://doi.org/10.1016/j.inffus.2019.08.004.
13. B. Li, Y. T. Hou, J. Liu, G. D. Nguyen and D. Taieb, Energy constraints and lifetime performance in wireless sensor networks, Mobile Networks and Applications, Vol. 10, No. 6, pp. 807-809, 2005.

14. D. K. Jagriti, Energy consumption reduction in S-MAC protocol for wireless sensor network, Procedia Computer Science, Vol. 143, pp. 757-764, 2018.

15. S. Li, Z. Huang and Z. Zeng, Dynamic simulation and research of routing protocol for mobile ad-hoc network, Simulation, Vol. 12, pp. 1-4, 2010.

16. S. S. Kumar and J. T. Thirukrishna, An efficient QoS based data packet transmission in wireless sensor networks using OREA, Wireless Personal Communication, 2020. https://doi.org/10.1007/ s11277-020-07295-1.

17. W. Liu, C. Zhang, G. Yao and Y. Fang, Delar: a Device-energyload aware relaying framework for heterogeneous mobile ad hoc networks, JSAC, Vol. 29, No. 8, pp. 1572-1584, 2011.

18. W. Mardini, M. B. Yassein, Y. Khamayseh and B. A. Ghaleb, Rotated hybrid, energy-efficient and distributed (r-heed) clustering protocol in wsn, red Wseas, Transactions on Communications, Vol. 13, pp. 275, 2014.

19. H. Kaufmann and R. Küng, A wireless data modem for local communications, in: Wireless Personal Communications. The Kluwer International Series in Engineering and Computer Science (Communications and Information Theory), M.J. Feuerstein and T.S. Rappaport (eds.), Vol. 197,Springer, Boston, 1993.

20. S. S. Kumar and J. T. Thirukrishna, OREA for improving data packet transmission in wireless sensor networks with cloud security mechanism, International Journal of Cloud Computing, Vol. 9, No. 2/3, pp. 245-257, 2020. https://doi.org/10.1504/IJCC.2020. 109379.

21. S. A. Sert, H. Bagci and A. Yazici, MOFCA: multi-objective fuzzy clustering algorithm for wireless sensor network, Applied Soft Computing, Vol. 30, pp. 151-165, 2015.

22. L. Miao, K. D. Djouani, G. Noel and A. M. Kurien, Network coding and competitive approach for gradient based routing in wireless sensor networks, Ad Hoc Networks, Vol. 10, No. 6, pp. 990-1008, 2012.

23. S. R. S. Krishna, S. Policherla, S. Srikanth, V. Raghu and J. T. Thirukrishna, Detecting CORONA VIRUS using chest X-ray images, International Journal of Advance Research and Innovative Ideas in Education, Vol. 7, No. 2, pp. 440-445, 2021.

24. D. Zhang, G. Li, K. Zheng, X. Ming and Z. H. Pan, An energy-balanced routing method based on forward-aware factor for wireless sensor networks, IEEE Transactions on Industrial Informatics, Vol. 10, No. 1, pp. 766-773, 2014.

25. C. Yawut, B. Paillassa and R. Dhaou, Mobility metrics evaluation for self-adaptive protocols, Journal of Networks, Vol. 3, No. 1, pp. 53-64, 2008.

26. J. T. Thirukrishna, M. V. Aishwarya, S. Mansi, B. Mounisha and K. Naksha, Efficient data Transmission in WSN using wearable sensors for Healthcare Monitoring, International Journal of Advance Research and Innovative Ideas in Education, Vol. 7, No. 2, pp. 446-457, 2021.

27. A. M. El-Bendary, WPAN-bluetooth simulation scenarios using block codes, in: Wireless Personal Communications, Signals and Communication Technology. R. Prasad (ed.),Springer, Singapore, 2018.

28. A. Capone, M. Pióro, Y. Li and D. Yuan, On packet transmission scheduling for min-max delay and energy consumption in wireless mesh sensor networks, Electronic Notes in Discrete Mathematics, Vol. 52, pp. 61-68, 2016.

Publisher's Note Springer Nature remains neutral with regard to jurisdictional claims in published maps and institutional affiliations. 


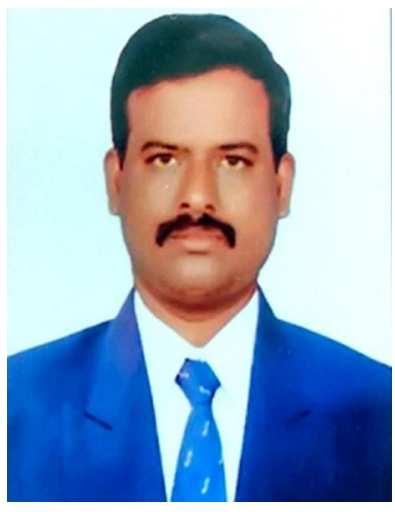

Senthil Kumar Swami Durai M.E., Ph.D., is pursuing Post-Doctoral Fellowship (PDF) Lincoln University College, Malaysia. He had completed $\mathrm{PhD}$ in Computer Science and Engineering in 2016, Chennai. He is presently working as Assistant Professor in the Department of Computer Science and Engineering, School of Engineering at Presidency University, Bengaluru, Karnataka, India. His main research interests are Sensor Networks, Big Data, and Network Security etc. He had published more than 12 Research articles in reputed journals.

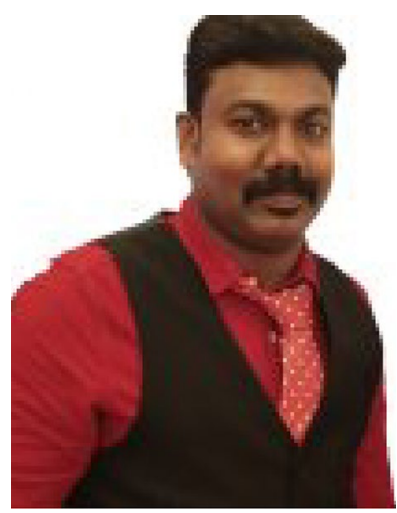

Balaganesh Duraisamy is presently working as Dean, Faculty of Computer Science and Multimedia at Lincoln University College, Malaysia. His main research interests are Web Mining. He had published more than 10 Research articles in the reputed journals.

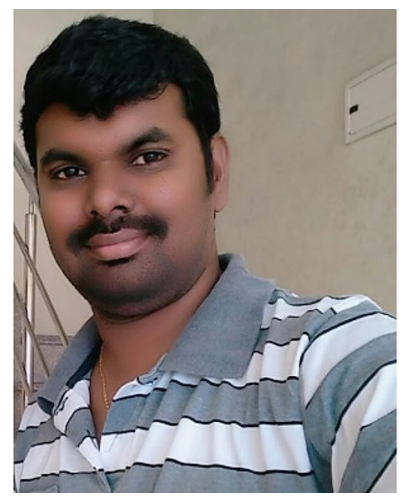

J. T. Thirukrishna received the M.E. degree in Computer Science and Engineering (First Class with Distinction) from Sona College of Technology, Salem, Tamil Nadu, India in 2010 and the Ph.D. degree in Information Communication and Engineering (CSE) at Anna University, Chennai, Tamil Nadu, India. Since 2020, he has been an Associate Professor with the Information Science and Engineering Department, Dayananda Sagar Institutions, Bangalore, India. $\mathrm{He}$ is the author of more than 15 articles, patent and book. His research interests include Wireless Sensor Networks, Data Science and Artificial Intelligence. He is a Journal Reviewer of SCI/SCIE/Web of Science/Scopus indexed Journals. He has applied many funding proposals to DST, CSIR and AICTE etc. Dr. J.T.Thirukrishna was a recipient of "Award of Excellence in Research Award 2020-2021" from Novel Research Academy and Global Teacher Award 2019 for Excellence, and the Senior member of IEEE, Life Member of ISTE and Cryptology Research Society of India. 